\title{
A boundary delimitation algorithm to approximate cell soma volumes of bipolar cells from topographical data obtained by scanning probe microscopy
}

\author{
Patrick Happel ${ }^{* 1,2}$, Kerstin Möller2,3, Ralf Kunz¹ and Irmgard D Dietzel2
}

\begin{abstract}
Background: Cell volume determination plays a pivotal role in the investigation of the biophysical mechanisms underlying various cellular processes. Whereas light microscopy in principle enables one to obtain three dimensional data, the reconstruction of cell volume from $z$-stacks is a time consuming procedure. Thus, three dimensional topographic representations of cells are easier to obtain by scanning probe microscopical measurements.

Results: We present a method of separating the cell soma volume of bipolar cells in adherent cell cultures from the contributions of the cell processes from data obtained by scanning ion conductance microscopy. Soma volume changes between successive scans obtained from the same cell can then be computed even if the cell is changing its position within the observed area. We demonstrate that the estimation of the cell volume on the basis of the width and the length of a cell may lead to erroneous determination of cell volume changes.

Conclusions: We provide a new algorithm to repeatedly determine single cell soma volume and thus to quantify cell volume changes during cell movements occuring over a time range of hours.
\end{abstract}

\section{Background}

Cell volume regulation occurs in a wide variety of tissues from kidney to brain [1-4]. Although much is known about ion and water fluxes involved in many regulatory processes, no method has so far been designed to investigate potential volume changes in moving cells. Light microscopy enables one to estimate the cellular volume via different techniques, ranging from extrapolation on the basis of the width and length of the cell [5], changes in light intensity and light scattering [6,7], various staining techniques $[8,9]$ to quantitative phase microscopy [10]. All these techniques fail when it is required to investigate the volume of a cell undergoing notable changes in shape such as occur during cell migration $[11,12]$ since they require constant parameters such as height or refractive index and some have additional disadvantages such as bleaching of the dye [13].

\footnotetext{
* Correspondence: patrick.happel@rub.de

${ }^{1}$ Central Unit for lonbeams and Radionuclides (RUBION), Ruhr University of Bochum, D-44780 Bochum, Germany

Full list of author information is available at the end of the article
}

A promising approach to circumvent these problems is to measure volume directly with a scanning probe microscope. Direct measurements of cellular volume have been performed by scanning ion conductance microscopy (SICM) on cellular layers [14] and single cells $[15,16]$ and by atomic force microscopy (AFM) on living and fixed cells $[17,18]$. The volume determined by SICM of cells forming a confluent layer has been validated by scanning confocal laser microscopy [14].

Volume determination by scanning probe microscopy assumes that cells are closely attached to the substrate and is mostly based on the height of every observed pixel $[14,15,17,18]$. When trying to investigate the volume dynamics of the somata of bipolar cells such as oligodendrocyte precursor cells (OPCs), the dimensions as well as the lateral resolution of the scan have to be restricted in order to obtain an acceptable temporal resolution. For the investigation of neural cells exhibiting long extensions most scanning frames inevitably crop the extended cellular ramifications. This leads to errors in the volume 
determination of migrating cells since the fraction of the processes located within the scanning frame may vary in successively obtained recordings, as for the process located on the right side in Figure 1.

We have previously proposed a method for distinguishing between cellular somata and processes during investigations of the surface of oligodendrocyte cell bodies at different developmental stages [19]. Here, every pixel exceeding a certain height was assigned to the cell soma. In cells undergoing marked changes in shape this method fails since it would result in different soma volumes if a cell flattens but performs a compensatory widening thus maintaining its volume. Hence, to estimate volume changes of single bipolar cell somata changing their shape and position we have now developed a novel procedure that allows us to separate the cell soma volume from the extended peripheral membrane processes of bipolar cells.

\section{Results and Discussion}

The boundary delimitation algorithm (BDA) for approximating the basal area of the cell soma of bipolar cells was divided into four steps as depicted in Figure 2. OPCs in

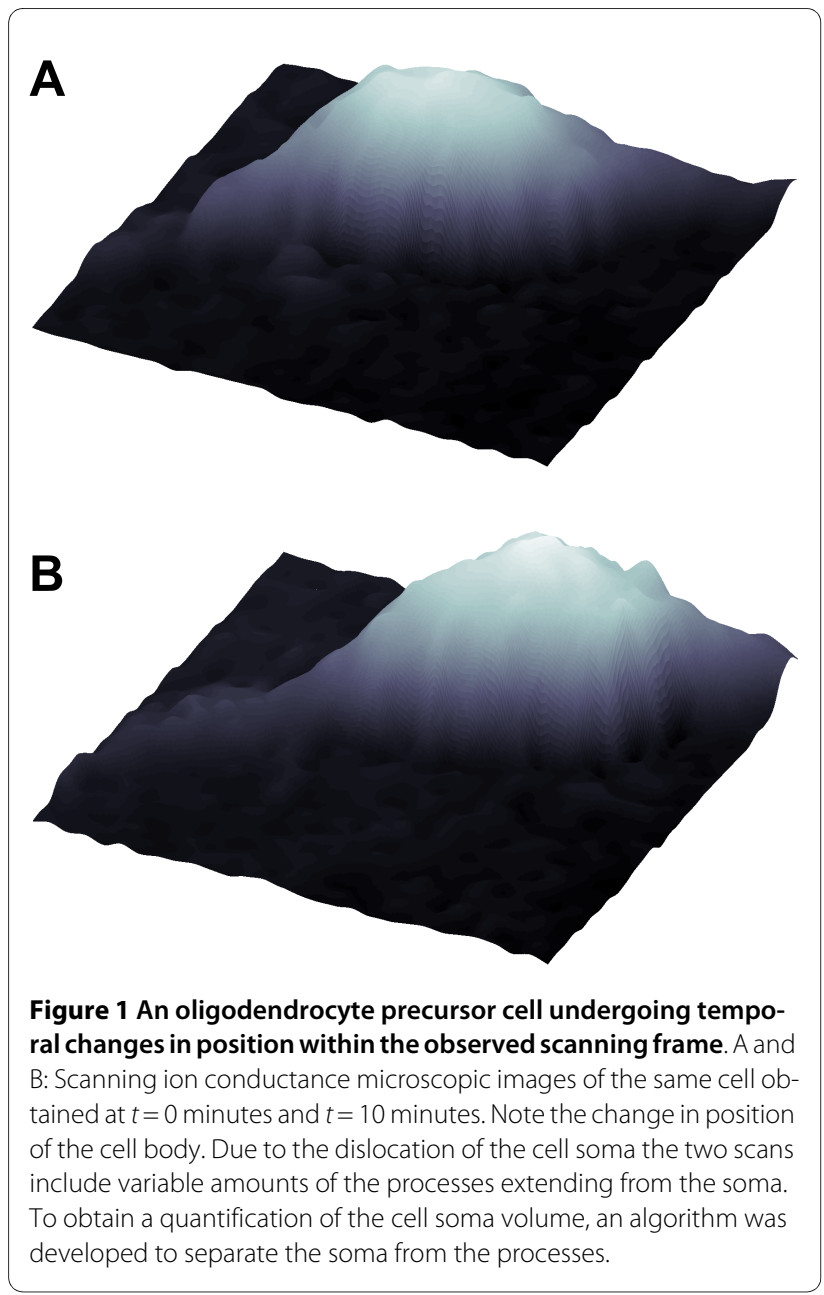

culture generally have two long processes at opposite poles of an ellipsoidal soma, and move in the direction of one of them. Whereas a single image does not allow the identification of the direction of movement it still allows the determination of the direction of the processes. We call this the "heading direction" of the cell. As the first step of the BDA the heading direction of the bipolar cell, indicated by the angle drawn in Figure 2A, was estimated and subsequently the cell rotated in order to position the heading direction of the cell parallel to the abscissa (Figure $2 \mathrm{~B}$ ). Second, the cell was divided into its front and rear parts at the level of the nucleus. Third, starting at the nucleus, the contour of the soma was approximated by linewise (as indicated by the dashed lines in Figure 2B) fitting of polynomials to the data for the frontal and the rear parts of the cell separately. The root of the fit for every single line, indicated by the red dot in Figure $2 \mathrm{C}$, was used to delimit the cell soma from the cell processes (Figure 2D). A compressed archive of the Matlab functions used to perform the BDA as detailed in the following is available as Additional File 1.

\section{Approximation of the position of the nucleus}

Atomic force microscopy measurements on hippocampal neurons revealed that the higher parts of the cell body form a harder structure and correspond most likely to the nucleus [20]. In order to determine a single point that represents the location of the nucleus the following procedure was employed: We stained the nucleus using Hoechst 33342 dye and recorded an epifluorescence as well as a phase contrast image.

Subsequently an SICM scan was performed and the relative position of the SICM scan was determined within the micrograph [19]. We then investigated the distance of the centroid of different horizontal sections through the SICM scans to the centroid of the Hoechst-stained nucleus. The horizontal sections consisted of the areas that were covered by pixels $P_{i}=\left(x_{i}, y_{i}, z_{i}\right)$ (with $i$ denoting the number of the pixel) exceeding a certain height $T$ $z_{\max }$, where $T$ denotes a predefined threshold and $z_{\max }$ denotes the maximum cell height. To calculate the position of the centroid $C_{T}$ we reduced the $z$-coordinates of $P_{i}$ to boolean values $z_{T, i}=\left[z_{i}>T z_{\text {max }}\right]$. The square brackets indicate a Heaviside-like function that yields 1 if the enclosed condition is true and 0 otherwise [21,22]. Furthermore, we assumed constant step sizes between the pixels and thus calculated the $x$-coordinate of $C_{T}, x_{C_{T}}$, as:

$$
x_{C_{T}}=\frac{\sum_{i} x_{i} \times z_{T, i}}{\sum_{i} z_{T, i}}
$$




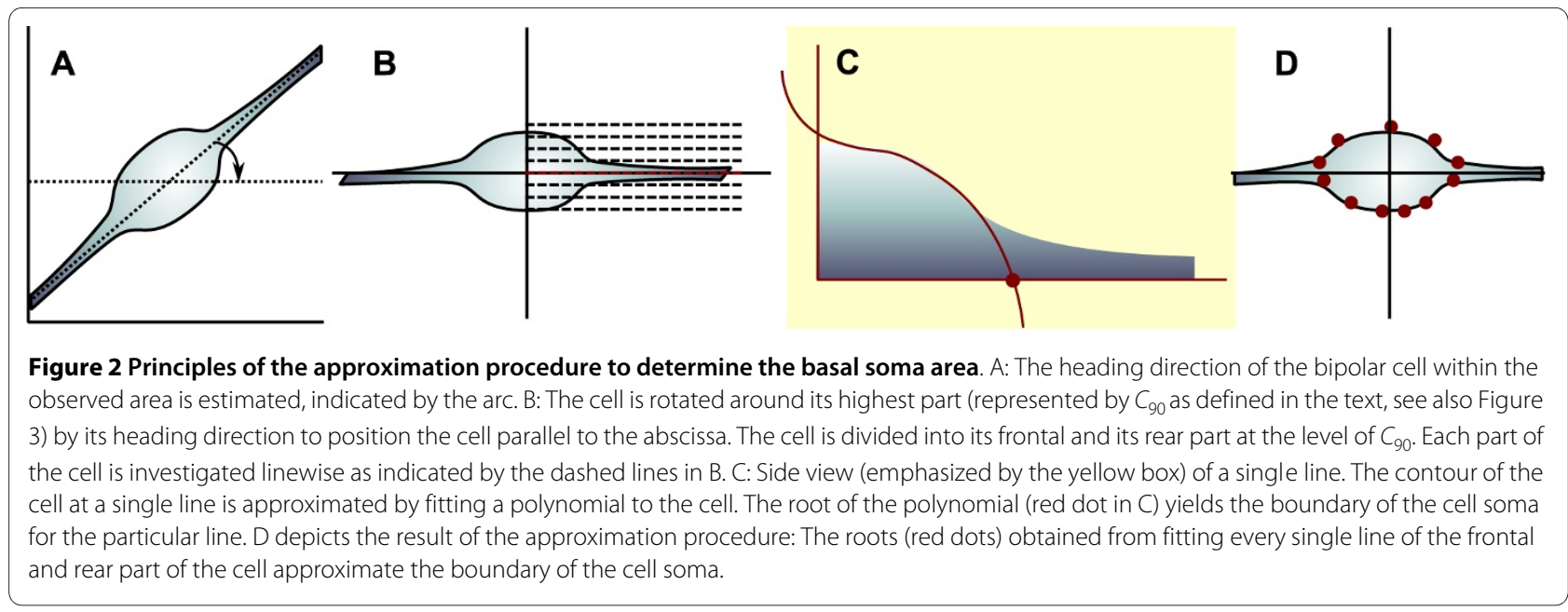

$y_{C_{T}}$ was calculated in the same manner.

We next investigated the distance between $C_{T}$ and the centroid of the Hoechst 33342 staining of the nucleus (see Methods section) for various thresholds $T$.

Figure 3A-C show the phase contrast, epifluorescence and SICM image of an OPC. The position of the SICM scan within the light microscopic image is depicted as the black square in Figure 3A. The positions of $C_{T}$ for $T=0.1$, $0.15, \ldots, 0.9$ and the centroid of the nucleus obtained from the epifluorescence staining $\left(C_{\text {fluo }}\right)$ are depicted in Figure
3D. $C_{90}$ (note that we use $T$ in percent when indexing or labeling, thus $C_{T=0.9} \equiv C_{90}$ ) exhibited the minimal distance to $C_{\text {fluo }}$. Figure $3 \mathrm{E}$ shows the average distances between $C_{T}$ and $C_{\text {fluo }}$ obtained from three different recordings. This confirms that $C_{90}$ is located closest to $C_{\text {fluo }}$. Note that representations determined by using a larger threshold such as $C_{95}$ often base on disjunct areas and were not investigated in detail. Thus we used $C_{90}$ to approximate the position of the nucleus in the following.

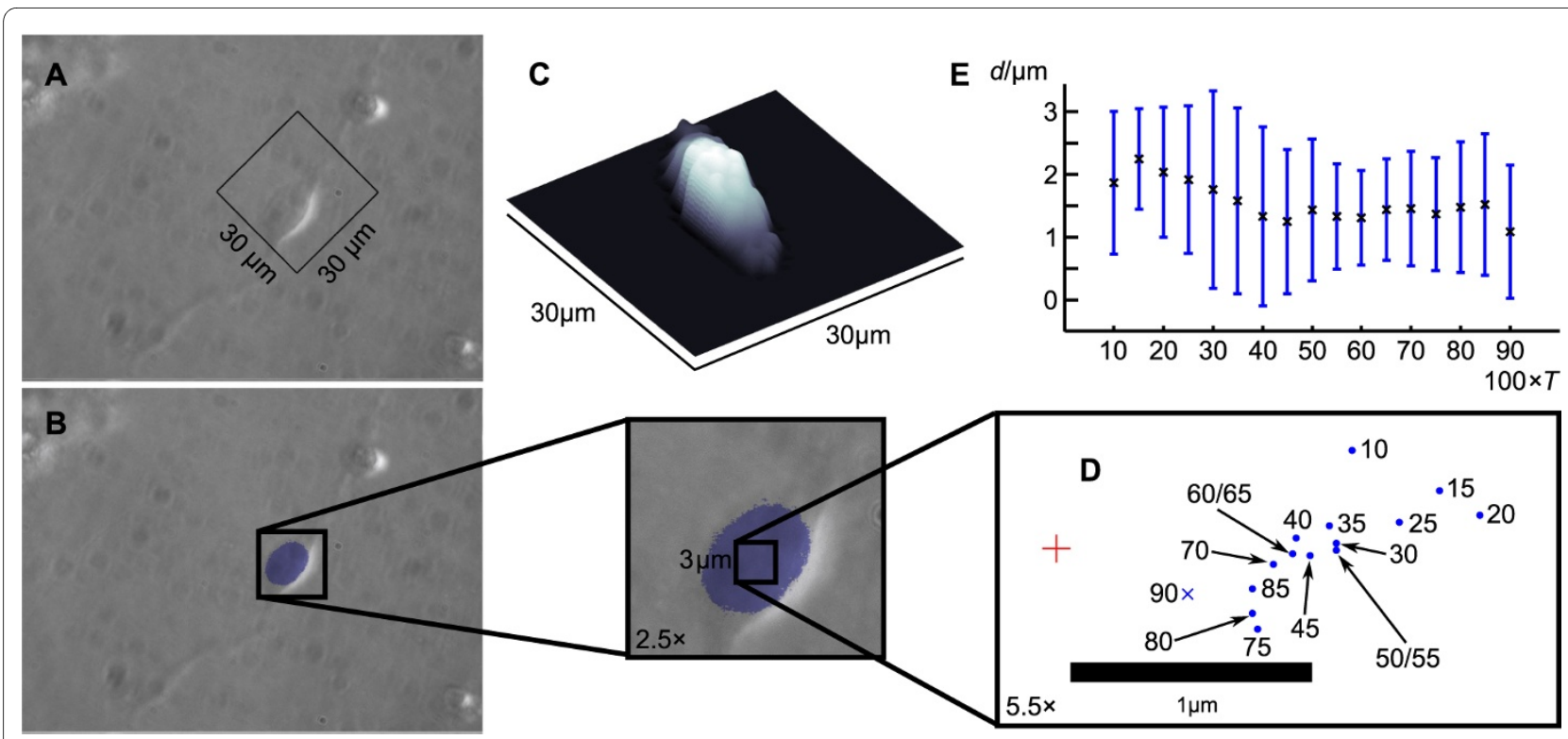

Figure 3 Representation of the location of the nucleus by $C_{90}$. A and B show light microscopic images from an oligodendrocyte precursor cell whose nucleus was stained using Hoechst 33342 (B) and that was scanned by backstep SICM (C). The position of the scan is depicted in A and a three dimensional representation of the data obtained by SICM is shown in C. The positions of $C_{T}$ for varying $T$ (between $10 \%$ and $90 \%$ of the maximal zvalue) calculated from the SICM data with respect to the position of the centroid of the stained area (obtained from fluorescence microscopy as shown in $B$, marked by the red cross-hair) are drawn rotated and magnified in D (blue dots and blue cross, labels indicate $T$ in percent). E shows the average distances between $C_{T}$ and the centroid of the staining of the nucleus obtained from 3 different determinations, error bars indicate \pm SD. 


\section{Estimation of the heading direction of the cell}

OPCs display a bipolar phenotype terminating in two cell processes that are most commonly originating from the opposite ends of the cell soma. This enables one to approximate the heading direction $\theta_{\mathrm{h}}$ of an OPC by rotating a straight line

$$
y(x, \theta)=x \tan \theta+y_{C_{90}}-x_{C_{90}} \tan \theta
$$

through $C_{90}$ as the approximation of a straight line through the nucleus. In order to determine the heading direction of the cell we considered the arcs from each pixel representing the cell to $y(x, \theta)$ Let $\phi_{i}(\theta)$ denote the smallest angle between $P_{i}$ and $y(x, \theta)$ and $r_{i}$ denote the distance from $C_{90}$ to $P_{i}$. Then the length $s_{i}(\theta)$ of the corresponding arc is calculated as $s_{i}(\theta)=\phi_{i}(\theta) r_{i}$. Figure $4 \mathrm{~A}$ illustrates the relations between the introduced angles, lines and points for two different pixels $P_{i}$ located at opposite sides of $C_{90}$. We now defined the angle $\theta_{\mathrm{h}}$, that minimized the sum of $s_{i}(\theta)$ and thus matched the condition

$$
\sum_{i}\left(s_{i}\left(\theta_{\mathrm{h}}\right) \times\left[z_{i}>1 \mu \mathrm{m}\right]\right)=\min
$$

as the heading direction of the cell. Here we assumed that pixels that exhibited a height of $\leq 1 \mu \mathrm{m}$ represented the cell culture dish rather than the cell. Equation (3) was solved numerically by testing all angles $0 \leq \theta \leq \pi$ in steps of $\Delta \theta=2 \pi / 360$.

\section{Rotating and interpolating the data}

After determining the heading direction of the cell data were rotated in order to position the cell parallel to the abscissa and translated such that $C_{90}$ was shifted into the origin of the new coordinate system. We denote the axes of the new coordinate system as $x^{\prime}-, y^{\prime}$ - and $z^{\prime}$ - axes and a rotated and translated pixel as $Q_{j}=\left(x_{j}^{\prime}, y_{j}^{\prime}, z_{j}^{\prime}\right)$, with $j$ indicating the number of the pixel in the rotated scan. To determine the lateral extent of the rotated scan we considered the distances of the vertices of the original scan and $y\left(x, \theta_{\mathrm{h}}\right)$ or a straight line through $C_{90}$ perpendicular to $y\left(x, \theta_{\mathrm{h}}\right)$ as illustrated in Figure $4 \mathrm{C}$. Since the approximation of the single line boundaries of the cell soma required lines of data points parallel to the heading direction of the cell, we defined the grid consisting of the projections $Q_{j}^{*}$ of $Q_{j}$ to the $x^{\prime}, y^{\prime}$ plane of the rotated and translated scan such that

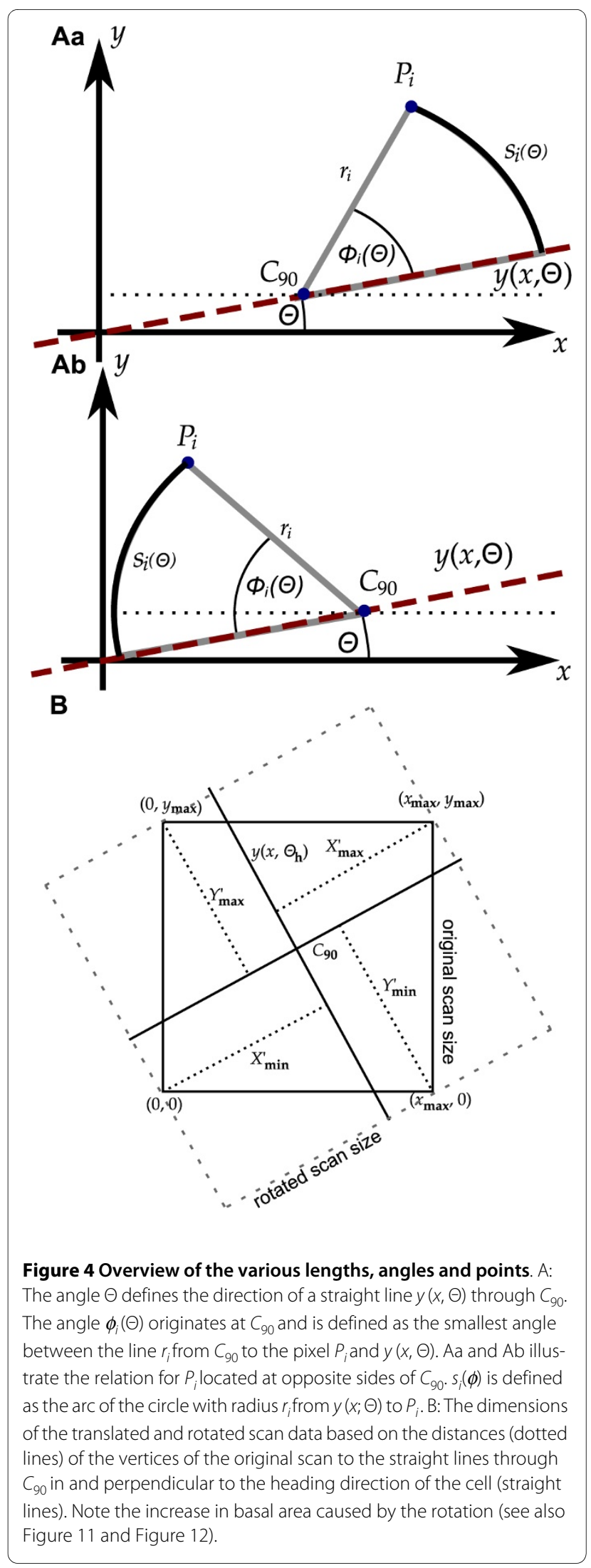




$$
\begin{array}{r}
x_{j}^{\prime} \in\left\{x_{\text {min }}^{\prime}, x_{\text {min }}^{\prime}+\Delta x, x_{\text {min }}^{\prime}+2 \Delta x_{,} \ldots,\right. \\
\left.x_{\min }^{\prime}+\left\lceil\left(X_{\min }^{\prime}+X_{\max }^{\prime}\right) / \Delta x\right\rceil \Delta x\right\}
\end{array}
$$

and

$$
\begin{gathered}
y_{j}^{\prime} \in\left\{\left[-Y_{\min }^{\prime} / \Delta y\right\rfloor \Delta y_{,}, \ldots,-2 \Delta y,-\Delta y, 0,\right. \\
\left.\Delta y, 2 \Delta y, \ldots,\left[Y_{\max }^{\prime} / \Delta y\right] \Delta y\right\} .
\end{gathered}
$$

Here $x_{\min }^{\prime}=\left(-X_{\min }^{\prime}\right)$ is the negative representation of the length $X_{\min }^{\prime}$ as a coordinate, $\Delta x$ and $\Delta y$ denote the step sizes of the original scan in the $x$ - and $y$-directions, respectively, and the truncated square brackets represent the ceil and the floor functions $[22,23]$.

To obtain the $z^{\prime}$ - coordinate of a pixel $Q_{j}$ we rotated its projection $Q_{j}^{*}$ into the original scan dataset by applying the inverse rotation matrix

$$
\mathbf{R}^{-1}=\left(\begin{array}{ll}
\cos \theta_{\mathrm{h}} & \sin \theta_{\mathrm{h}} \\
-\sin \theta_{\mathrm{h}} & \cos \theta_{\mathrm{h}}
\end{array}\right)
$$

and subsequently re-translated it by $\left(-x_{C_{90}},-\gamma_{C_{90}}\right)$. We refer to the resulting projection as $Q_{j, \text { trans }}^{*}$. If $Q_{j, \text { trans }}^{*}$ was located outside the original scan, we defined $Q_{j}=\left(x_{j}^{\prime}, y_{j}^{\prime}, 0\right)$. Otherwise we considered the four projections $R_{1, \ldots, 4}^{*} \in\left\{P_{i}^{*}\right\}$ (here $P_{i}^{*}$ denotes the projection of $P_{i}$ to the $x$-, $y$-plane) that surrounded $Q_{j, \text { trans }}^{*}$ as depicted in Figure 5B. The $z$-coordinates of the corresponding pixels were known from the original data. Each set of three out of these four projections defines a triangle as indicated by the dotted lines in Figure 5B. In the following we refer to the four triangles as $\mathbf{M}_{k}$ (with $k=1,2,3,4$ ) and to the vertices of one triangle as $M_{k, 1}^{*}$ with $l=1,2,3$. We selected $l$ such that the right angle was located at $M_{k, 1}^{*}$ and furthermore such that $\gamma_{M_{k, 1}^{*}}=y_{M_{k, 2}^{*}}$ and $x_{M_{k, 1}^{*}}=x_{M_{k, 3}^{*}}$. An example is shown in Figure 5C. If and only if $Q_{j, \text { trans }}^{*}$ was located inside $\mathbf{M}_{k}$ the sum $\zeta_{k}$ of the angles at $Q_{j, \text { trans }}^{*}$ to the vertices of $\mathbf{M}_{k}$ amounted to $2 \pi$ [24].

We next considered the plane defined by the pixels $M_{k, l}$ that corresponded to the projections $M_{k, 1}^{*}$. The $z$-value $z_{k}(x, y)$ of this plane at a position $(x, y)$ is given by

$$
\begin{array}{r}
z_{k}(x, y)=z_{M_{k, 1}}+\frac{\left(x-x_{M_{k, 1}}\right)\left(z_{\left.M_{k, 2}-z_{M_{k, 1}}\right)}\right.}{\Delta x} \\
+\frac{\left(y-y_{M_{k, 1}}\right)\left(z_{M_{k, 3}}-z_{M_{k, 1}}\right)}{\Delta y} .
\end{array}
$$

We now interpolated $z_{j}^{\prime}$ as the average of $z_{k}\left(Q_{j, \text { trans }}^{*}\right)$ if $Q_{j, \text { trans }}^{*}$ was located inside $\mathbf{M}_{\mathrm{k}}$ :

$$
z_{j}^{\prime}=\frac{\sum_{k=1}^{4}\left(z_{k}\left(Q_{j, \text { trans }}^{*}\right) \times\left[\zeta_{k}=2 \pi\right]\right)}{\sum_{k=1}^{4}\left[\zeta_{k}=2 \pi\right]} .
$$

\section{Approximation of the contour of a single data row}

To trace the contour of the cell soma and thus to crop the processes we now considered every data row (all data points with the same $y$ ) separately. The corresponding $y^{\prime}$ values were defined by equation (4). Figure 6 shows sketches of the contours of two characteristic cell shapes; an almost circular cell body that is easy to distinguish from the cell processes (Figure 6A) and a cell soma that protruded into the direction of one of the extensions (Figure 6B). Thus, as indicated in Figure 6B, we assumed that a polynomial of third degree was convenient to approximate the cell soma contour but still suitable to crop the cell process.

To approximate both ends of the cell within a single data row at a fixed $y^{\prime}$-level we subdivided the data into positive and negative, or frontal and rear, parts with respect to the corresponding $x^{\prime}$-coordinates. In the following we describe the fitting procedure for the positive part, thus $x^{\prime}>0 . S_{j}^{+}=\left(x_{j}^{\prime}, z_{j}^{\prime}\right)$ was defined as the projection of $Q_{j}$ to the $x^{\prime}, z^{\prime}$-plane and furthermore $\mathbf{S}_{\gamma^{\prime}}=\left\{S_{p, \gamma^{\prime}}^{+}\right\}$with $p=0,1,2, \ldots$ as the set of projections at a constant $y^{\prime}$ such that for all $p>0$

$$
z_{S_{p, y^{\prime}}^{+}}^{\prime}>0 \wedge x_{S_{p, y^{\prime}}^{\prime}}^{\prime} \geq 0 \wedge x_{S_{p, y^{\prime}}^{\prime}}^{\prime}<x_{S_{p+1, y^{\prime}}^{\prime}}^{\prime} .
$$

Furthermore, we defined $S_{0, y^{\prime}}^{+}$such that $x_{S_{0, y^{\prime}}^{+}}=x_{S_{1, y^{\prime}}^{+}}-\Delta x$. This definition only included pixels with non zero $z^{\prime}$-coordinates (since the data points were filtered this is equivalent to $z^{\prime}>1 \mu \mathrm{m}$, see Methods section). In general $n+2$ data points are needed to fit a polynomial of $n$th degree $(n+1$ data points define the polynomial). Furthermore, we assumed that the cell body is represented by the data points whose $x^{\prime}$-coordinates are located close to zero. Thus we additionally tested whether 


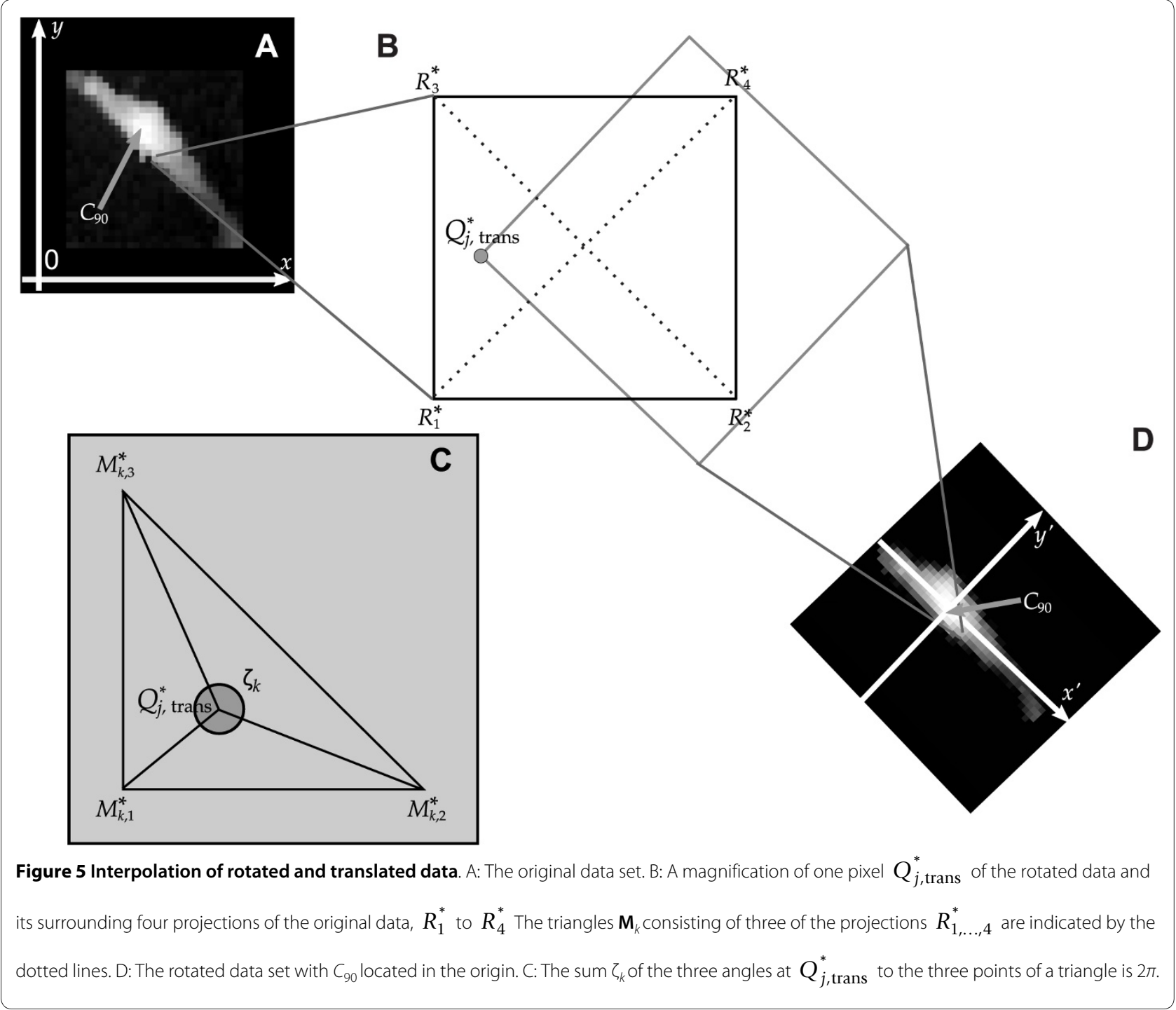

there was no gap within $S_{0, y^{\prime}}^{+}, \ldots, S_{n+1, y^{\prime}}^{+}$and it therefore matched the condition

$$
\begin{gathered}
x_{S_{p-1, y^{\prime}}^{+}}^{\prime}+\Delta x=x_{S_{p, y^{\prime}}^{+}}^{\prime}, \forall p \in\{1,2, \ldots, n+1\} \\
\quad \text { and } x_{S_{0, y^{\prime}}^{+}}^{c^{+}}<0 .
\end{gathered}
$$

Otherwise, data points with $x^{\prime}$-coordinates close to zero existed with $z^{\prime}=0$. This most likely occured at the borders of the cell soma in $\pm y^{\prime}$-direction and was treated as a special case described later in this section.

To fit a polynomial of $n$th degree to the data we used the function fit from Matlab's Curve Fitting Toolbox that implements a least square algorithm $[25,26]$. It provides, among others, the value $R_{\text {adj }}^{2}$ that represents the goodness of the fit considering the number of data points that were approximated by the fit. We investigated the goodness of the fits to an increasing number $r$ of data points. We refer to the subset of $\mathbf{S}_{y^{\prime}}$ that contains the first $r$ elements as $\mathbf{S}_{r, y^{\prime}}=\left\{S_{0, y^{\prime}}^{+}, \ldots, S_{r, y^{\prime}}^{+}\right\}$and we denote the goodness of the fit to $\mathbf{S}_{r, y^{\prime}}$ as $R_{\gamma^{\prime} \text {,adj }}^{2}(r)$ Additionally, we defined $X_{y^{\prime}}(r)$ to be the smallest, positive, non-complex root of the polynomial $F_{y^{\prime}}(r)=\sum_{m=0}^{n} A_{r, m} x^{\prime m}$ that was determined by the function fit. We approximated the polynomial boundary of the cell soma for each line segment towards the direction of fitting as the $X_{y}(r)$ that matched the condition

$$
R_{y^{\prime}, \text { adj }}^{2}(r) \times\left[X_{y^{\prime}}(r) \text { exists }\right]=\max
$$




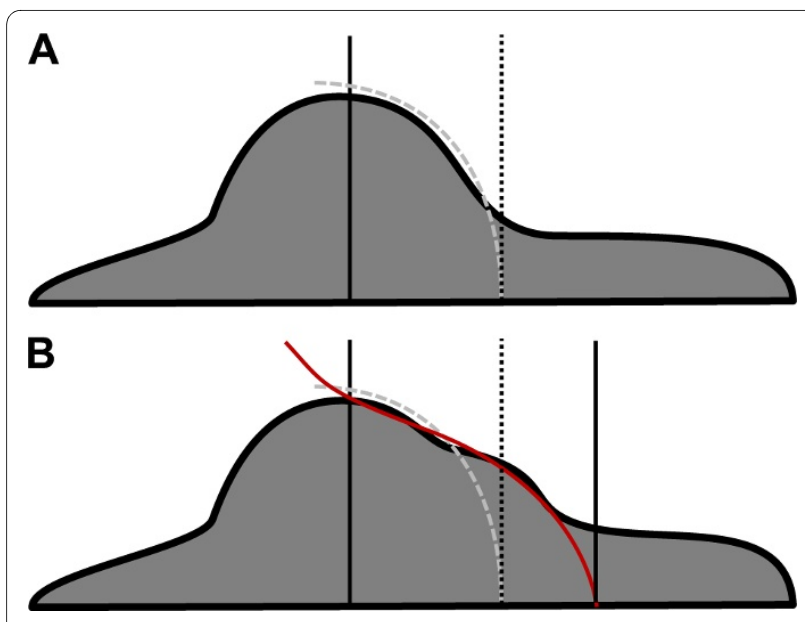

Figure 6 Characteristic contours of the soma of OPCs. A: Contour of a cell soma approximating a circular shape. The black line marks the level of $C_{90}$. The dashed gray line indicates a parabola fitted to the cell contour that traces the soma but crops the process. B: Contour of a cell soma protruding into the direction of a process. A parabola (gray dashed line) would crop the protrusion of the soma whereas a polynomial of third degree includes the protrusion but still crops the process.

with $r=n+1, n+2, \ldots, p_{\max }$. Here $p_{\max }$ denotes the largest index $p$ of the projections included in $\mathbf{S}_{y^{\prime}}$ Figure 7 shows examples of the fitting procedure for $r=4,8,9$ and 14 , respectively, with $n=3$, hence fitting polynomials of third degree. For $r=4$ and $r=14$ (Figure 7A and 7D) $F_{y}(r)$ had no real root with a corresponding positive $x^{\prime}$-coordinate, thus these fits were not taken into consideration.

Since $R_{y, \text { adj }}^{2}(r=8)>R_{y, \text { adj }}^{2}(r=9)$ (Figure 7B and 7C) $X_{y}(r$ $=8$ ) (indicated by the red arrow-head in Figure 7C) was used to approximate the cell soma boundary at the corresponding $y^{\prime}$-level. Note that the goodness of the fit to $\mathbf{S}_{8, y^{\prime}}$ was larger than those of all other fits that exhibited $X_{y^{\prime}}(r \neq$ 8 ) but are not shown in Figure 7 for clarity.

If the procedure failed to determine a cell soma boundary for the investigated set of data points $\mathbf{S}_{y^{\prime}}$ no $r$ with a corresponding $X_{y^{\prime}}(r)$ existed. We then defined the boundary to be $X_{y^{\prime}}(r=n)$, if it existed. Note that $R_{y^{\prime}, \text { adj }}^{2}(r=n)$ is not defined [26]. If $X_{y^{\prime}}(r=n)$ did also not exist we repeated the procedure with $n:=n-1$ as long as $n>1$, thus fitting polynomials of a reduced degree. In all cases investigated this procedure led to detection of bordering pixels.

Figure 8 summarizes the fitting procedure as described above in a flow chart. Due to space restrictions the chart omits the test of whether $R_{\gamma^{\prime}, \text { adj }}^{2}(r=n)$ existed as well as the test of whether $n>1$, indicated by the dotted arrow in the lower right part of the chart. This procedure was named fitBest.
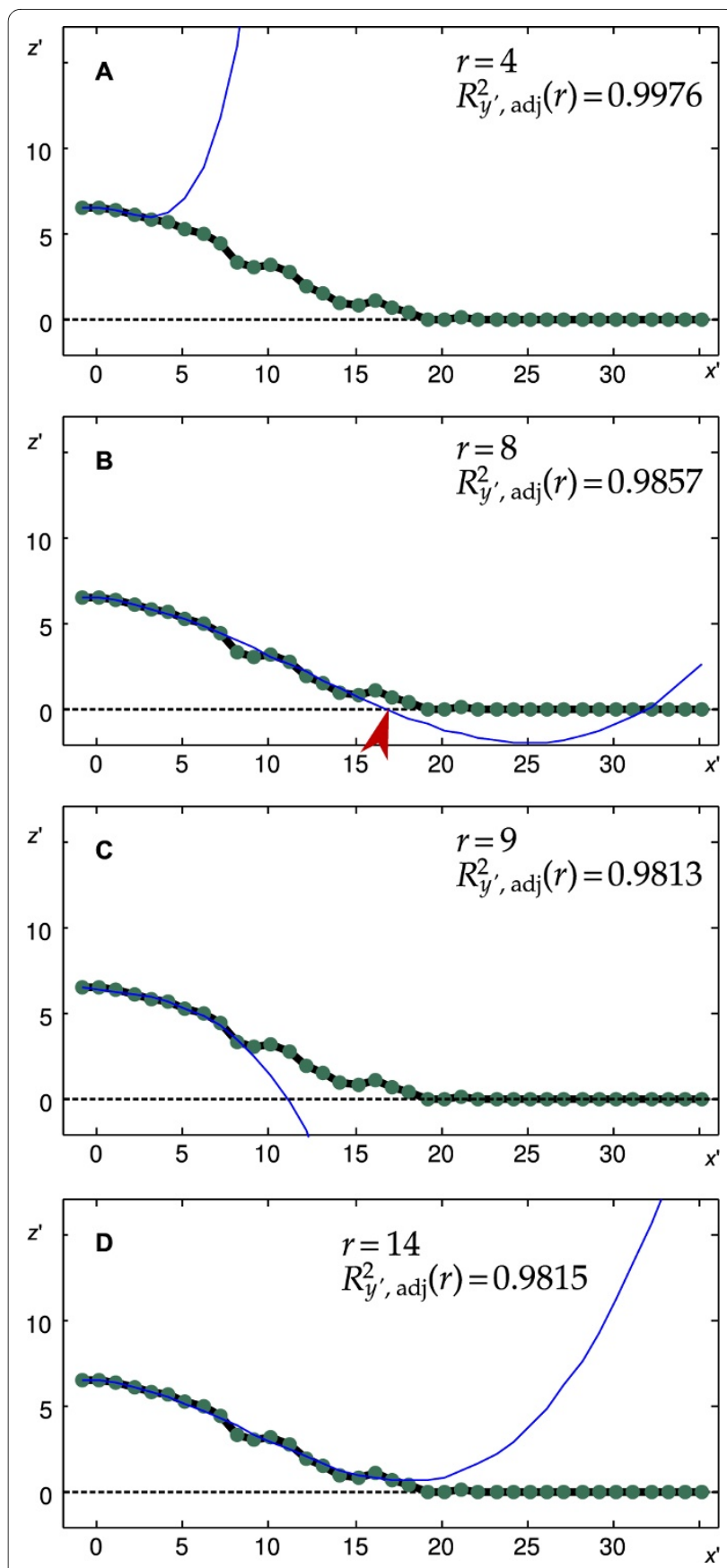

Figure 7 Example of the fitting procedure. A-D show the approximated polynomials $F_{y^{\prime}}(r)$ (blue lines) for $r=4 ; 5 ; 9$ and 14, respectively. Neither $F_{y^{\prime}}(r=4)$ nor $F_{y^{\prime}}(r=14)$ (panels $A$ and $\left.D\right)$ had a corresponding $X_{y^{\prime}}$ $(r)$ and thus were not taken into consideration. Both $F_{y^{\prime}}(r=8)$ and $F_{y^{\prime}}(r=$ 9) (panels $B$ and $C$ ) had a corresponding $X_{y^{\prime}}(r)$; thus the corresponding $R_{y^{\prime}, \text { adj }}^{2}(r)$ were compared. Since $R_{\gamma, \text { adj }}^{2}(r=8)>R_{y, \text { adj }}^{2}(r=9)$ $X_{y^{\prime}}(r=8)$ (red arrow-head in B) was selected as the boundary of the cell soma for the investigated $y^{\prime}$. 


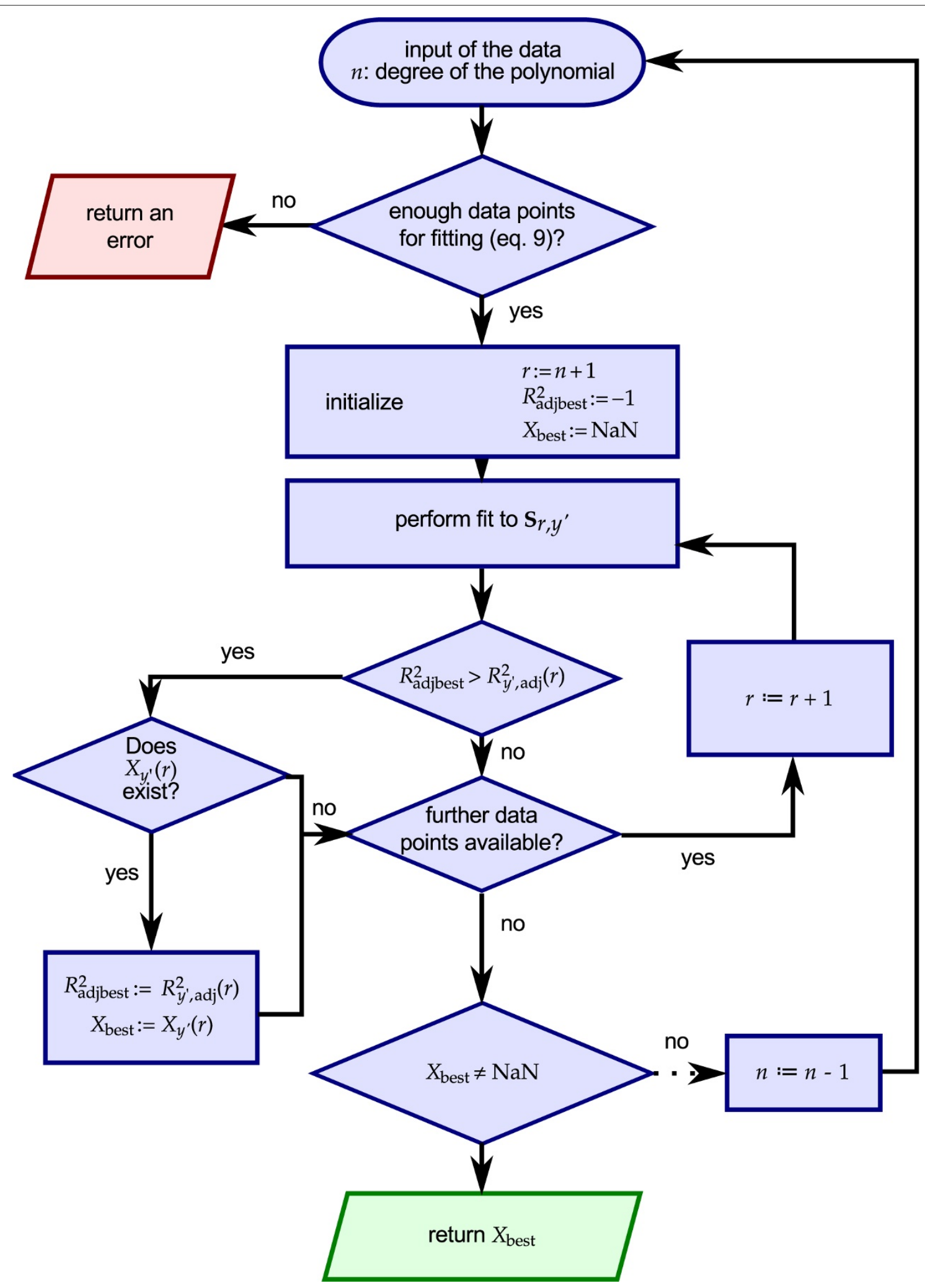

Figure 8 Flow-chart of the procedure to find the best fit. The procedure investigates the approximations to an increasing number of data points and selects the one with a positive, non-complex root and the best corresponding $R_{\gamma^{\prime} \text {, adj }}^{2}$ Note that the chart omits some additional tests (see text) to ensure an error free operation as indicated by the dotted arrow in the lower right part. Note that NaN $\equiv$ not a number. 


\section{Special cases of the fitting procedure}

As indicated in Figure 8 an error was returned if the investigated set of data points did not match the conditions listed in equation (9). In this case data points with a corresponding $z_{j}^{\prime}=0$ existed within the first $n+1$ data points in the fit direction. This most likely occurred at the borders of the cell soma in $\pm y^{\prime}$-direction. This special situation might occur under two conditions. In the first case the cell body approximates to a circular shape causing the boundary perpendicular to the direction of fitting to consist of only a few pixels. Furthermore, the number of pixels available to the fitting procedure as depicted in Figure 7 is decreased by the division of the cell into its frontal and its rear part. Secondly, OPCs in a later stage of development might exhibit small additional extensions that grow perpendicularly to the heading direction.

It was important to consider these cases in order to provide an errorless and thus automatic processing of the data. There are different strategies to determine the boundary of the cell soma at these locations depending either on the chosen degree of the polynomial fitted to $\mathbf{S}_{y^{\prime}}$ as well as whether potential extensions at these sides of the cell soma should be included or excluded from the soma approximation. The most restrictive and simple solution would be to omit and thus to crop these lines.

To obtain a more accurate fit and to include potential cell extensions at these sides we introduced three more functions: fitOnePoint, fitTwoPoints and fitThreePoints that were executed depending on the number of data points with $z^{\prime}>0$. We considered the set of pixels $\mathbf{T}_{y^{\prime}}=\left\{T_{0, y^{\prime}}^{+}, T_{1, y^{\prime}}^{+}, \ldots, T_{n+2, y^{\prime}}^{+}\right\}$that matched all conditions listed in equation (8) except one: The $z^{\prime}$-coordinate was not tested, thus $\mathbf{T}_{y^{\prime}}$ might also include projections with $z^{\prime}$ $=0$. Let $N=\sum_{m=1}^{n+1}\left[z_{T_{m, y^{\prime}}^{+}}^{\prime}>0\right]$ be the number of projections with a $z^{\prime}$-coordinate exceeding zero. If $N=4$ we executed the function fitBest. If $N=1, N=2$ or $N=3$ we executed the functions fitOnePoint, fitTwoPoints or fitThreePoints, respectively. Note that these functions might result in more than one boundary for the particular $y^{\prime}$ level, thus the resulting approximated cell soma might appear jagged.

The simplest case is $N=1$ and the corresponding function fitOnePoint. We refer to the non-zero data point as $T_{u, y^{\prime}}^{+}$and used the roots of a parabola through $T_{u-1, y^{\prime}}^{+}, T_{u, y^{\prime}}^{+}, T_{u+1, y^{\prime}}^{+}$as the boundary if $u<4$, otherwise the line was cropped.

Let the two non-zero projections be $T_{u, y^{\prime}}^{+}$and $T_{v, y^{\prime}}^{+}$ with $u<v$ in the case of $N=2$ (function fitTwoPoints). We first considered the case $v-u=1$, hence, the two points were neighbors. We fitted a polynomial of third degree to $T_{u-1, y^{\prime}}^{+}, T_{u, \gamma^{\prime}}^{+}, T_{v, y^{\prime}}^{+}$and $T_{v+1, y^{\prime}}^{+}$and used its roots as the boundary in this case except if $v=4$ and $z_{T_{5, y^{\prime}}^{\prime}}^{\prime}>0$. In the latter case we cropped the structure assuming that it did not belong to the cell soma.

If $v-u>1$ we only assigned $T_{u, y^{\prime}}^{+}$to the cell soma and approximated the contour of the cell soma by the roots of the parabola through $T_{u-1, \gamma^{\prime}}^{+}, T_{u, y^{\prime}}^{+}, T_{u+1, \gamma^{\prime}}^{+}$as in the function fitOnePoint.

The most complicated case was $N=3$. We refer to the single projection with zero $z^{\prime}$-coordinate as $T_{u, y^{\prime}}^{+}$In this case the approximation was performed differently for varying values of $u$. If $u=1$ we considered the $z^{\prime}$-coordinate of the projection $T_{5, y^{\prime}}^{+}$. If $z_{T_{5, y^{\prime}}^{+}}^{\prime}>0$ we assumed that the cell soma exhibited an asymmetric shape and applied the function fitBest. Otherwise, if $z_{T_{5, y^{\prime}}^{\prime}}^{\prime}=0$ we approximated the cell soma boundary for the particular $y^{\prime}$ by the roots of a polynomial of third degree fitted to $T_{1, \gamma^{\prime}}^{+}, \ldots, T_{5, y^{\prime}}^{+}$.

If $u \quad\{2,3\}$ we applied fitOnePoint to the single, nonzero projection and fitTwoPoints to the two neighboring non-zero projections, respectively. If $u=4$ we considered the $z^{\prime}$-coordinate of the first point opposite to the direction of fitting, $T_{0, y^{\prime}}^{+}$. If $z_{T_{0, y^{\prime}}^{\prime}}^{\prime}>0$ we applied fitBest, otherwise we approximated the cell soma boundary at the current $y^{\prime}$-level by the roots of a polynomial of third degree fitted to $T_{1, y^{\prime}}^{+}, \ldots, T_{4, y^{\prime}}^{+}$.

\section{Approximation of the volume of the cell soma}

To approximate the cell soma volume we summed the $z$ coordinates of every pixel located within the approximated boundaries of the cell soma. This required that the height of every pixel located within the approximated cell soma boundary was known. Hence, if a single delimitation of the cell soma was located outside the original scan we were not able to approximate the cell soma volume and the recording was discarded. This happened if the cell body was in part located outside of the SICM image or very close to its borders.

\section{Evaluation of the procedure}

To evaluate the BDA we simulated objects of known volume and applied the morphometric fitting procedure to investigate any potential effect of geometry on the volume determinations. We have previously determined the 
restrictions of scan size and resolution for the successful investigation of migrating OPCs [27]. In brief, to image migrating OPCs with a suitable frame rate using our present SICM the dimensions of the recordings had to be restricted to $30 \mu \mathrm{m}$ squares with a lateral step size of 1 $\mu \mathrm{m}$, limiting the SICM images to 900 pixels.

We first applied the BDA to a hemisphere with a radius of $r_{0}=5$ pixels (since the length of the cell body of an OPC is approximately $10 \mu \mathrm{m}$ ) in a data set consisting of 900 pixels as depicted in Figure 9A. The volume $V_{\text {comp }}$ computed by the BDA (omitting the determination of a heading direction as well as rotation and translation) was the same as the volume $V_{\text {sum }}$ calculated by summing the volume of the columns above each pixel.

We next compared the determination of the volume of an half-ellipsoid with the two methods. A possible effect of the direction of fitting was tested by applying the BDA to an ellipsoid defined by the three radii $r_{x}, r_{y}$ and $r_{z}$ with $r_{x}>\mathrm{r}_{\mathrm{y}}$ and vice versa, as depicted in Figure 9B and 9C (the corresponding radii are $r_{x}=0.8 r_{0}, r_{y}=1.25 r_{0}, r_{z}=r_{0}$ and $r_{x}$ $\left.=1.25 r_{0}, r_{y}=0.8 r_{0}, r_{z}=r_{0}\right)$. Again, no difference was found between $V_{\text {comp }}$ and $V_{\text {sum }}$.

To investigate whether the BDA in principle allows one to determine the volume of an object that flattens but maintains its volume by a compensatory widening we computed the volumes of an ellipsoid defined by the radii $r_{y}=r_{0}, r_{x}=t r_{0}$ and $r_{z}=r_{0} / t$ with $1 \leq t \leq 2$ in step sizes of $\Delta t$ $=0.05$. Figure $9 \mathrm{G}$ (blue crosses) shows the computed volume normalized to $V_{\text {sum }}$ for every investigated value of $t$. There is no difference between $V_{\text {comp }}$ and $V_{\text {sum }}$, thus $V_{n}=$ 1 . In contrast, the computed volume did not match $V_{\text {sum }}$ when it was determined by using the method that every pixel exceeding a predefined threshold was assigned to the cell soma $[16,19]$. The normalized volumes are displayed in Figure 9G (red dots and cross-hairs) for an absolute and a relative threshold. In the following we only consider the determination using a relative threshold since it is clearly visible that the use of an absolute threshold leads to increasing differences in the determination of the soma with increasing elongation of the ellipsoid. Additionally, we observed no difference in the volume
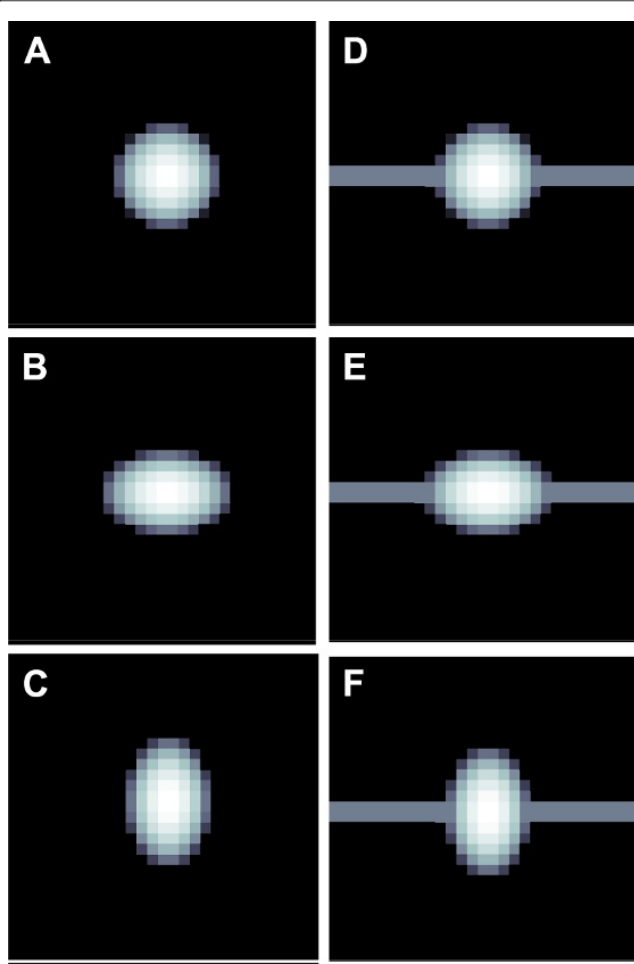

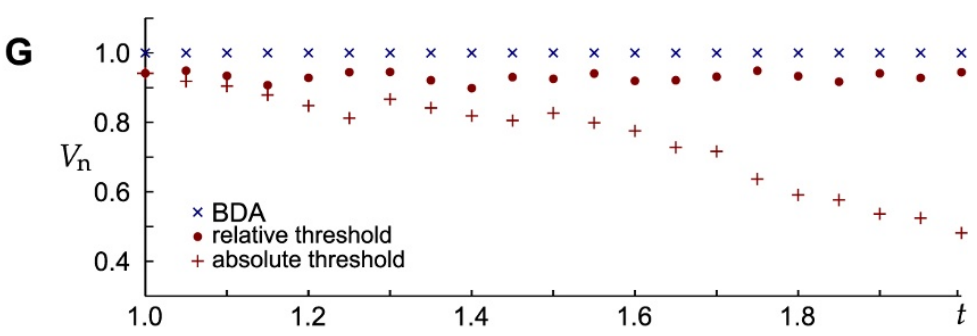

H $\quad V_{n}$

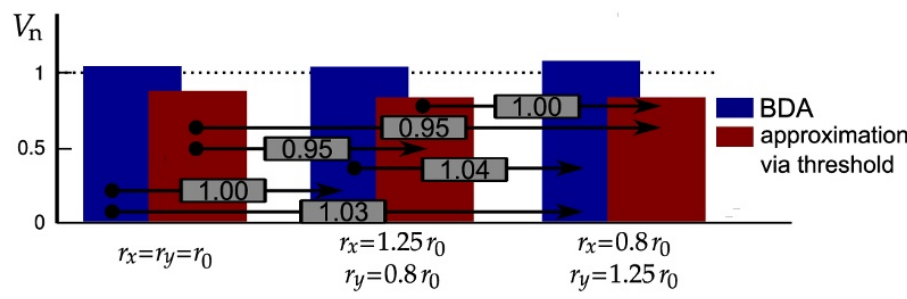

I

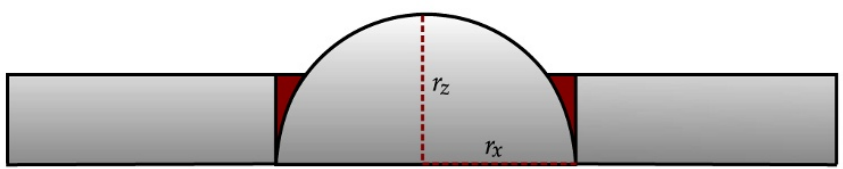

Figure 9 Application of boundary delimitation algorithm to simulated objects. A-C: Half-ellipsoids with the corresponding radii $r_{x}=r_{y}=r_{0}(A), r_{x}$ $=1.25 r_{0} ; r_{y}=0.8 r_{0}(B)$ and $r_{x}=0.8 r_{0} ; r_{y}=1.25 r_{0}(C)$. The radius in $z$-direction is $r_{z}=r_{0}$. D-F: Hemisphere/half-ellipsoids from A-C with additional extensions. G: Normalized volume $\left(V_{n}\right)$ computed by the boundary delimitation algorithm (BDA) as well as by thresholding simulating a half-ellipsoid with the radii $r_{x}=t r_{0}$ and $r_{z}=1 / t r_{0}$ for $1 \leq t \leq 2$ and $\Delta t=0.05$. Corresponding thresholds were $0.4 r_{z}$ and $0.4 r_{0}$, respectively. H: Volumes of the objects from D-F computed by the BDA (blue) and by thresholding (red) using a threshold of $0.4 r_{z}$. Gray boxes indicate erroneously determined changes in volume when the shape of the object changes as indicated by the respective arrows. I: The addition of the extensions changes the volume of the simulated cell soma with respect to the mere half ellipsoid as indicated by the red area. 
determined by the BDA and $V_{\text {sum }}$ when varying $r_{y}$ instead of $r_{x}$ or when varying both lateral radii by defining $r_{x}=r_{y}=$ $t^{1 / 2} r_{0}$.

To simulate a bipolar cell we added extensions in $\pm x^{\prime}$ direction to a hemisphere of radius $r_{0}$ as well as to the ellipsoids. Images of the resulting objects are depicted in Figure 9D-F. The height of the extension was chosen as $r_{0} / 2$ and its width as $2 r_{0} / 5$. Every $z$-value at the corresponding positions was adjusted to $r_{0} / 2$ if the $z$-value calculated by equation (11) (see Methods section) was below $r_{0} / 2$. This avoids a gap between the half-ellipsoid and the extension but also increases the $z$-value of some pixels of the half-ellipsoid such that the volume differs from the volume $V_{\text {sum }}$ computed by summing the $z$-values of the mere half-ellipsoid as depicted in Figure 9I. To our knowledge no exact definition exists describing where the cell soma ends and the cell process starts. At positions where the soma merges into the neurite a gradual decline of the soma and a corresponding increase of soma volume most likely occurs (Figure 9I).

Here we chose to use the calculated volume of the halfellipsoid without extension as reference. Since we calculated the soma volume by summing all $z$-values corresponding to pixels within the approximated soma boundary an overestimation of the soma volume at positions merging into the neurites (Figure 9I) could be induced by the BDA.

The approximated volume, normalized to $V_{\text {sum }}$ of the corresponding hemisphere or ellipsoid without extension, is shown in Figure 9H. As expected, the BDA (blue bars in Figure 9H) overestimates the volume with respect to $V_{\text {sum. }}$. In contrast, the approximation via the threshold method [16,19], in this case applied using a threshold of $r_{0} / 2$, underestimates the volume with respect to $V_{\text {sum }}$ since it omits all sections of the ellipsoid with a height below the selected threshold. Putative cell shape changes as depicted in Figure 9E-G would result in detections of relative soma volumes as indicated in the gray boxes in Figure 9H. Erroneous changes due to different shapes are indicated by the arrows. Both methods lead to almost similar errors (about 5\%) in the determination of soma volume changes.

Since it is unlikely that the shape of the soma changes while the extensions maintain their shape we next investigated the impact of changes in the shape of the extensions. Figure 10A shows the volume determined by the BDA when applied to a hemisphere with adjacent extensions (as depicted in Figure 9E) of varying relative height $h r_{0}$, normalized to $V_{\text {sum }}$ of the hemisphere without extensions. As expected from the result shown in Figure 9H, our method overestimates the volume with increasing height. An increase in the height of the extension from $h$
$=0.2$ to $h=0.6$ results in an erroneous detection of a soma volume increase of about $6 \%$. Although the relation seems to be linear in the depicted range, it is more complex: A threefold increase in the height of the extension from $h=0.3$ to $h=0.9$ leads to an erroneous detection of a volume increase of about $9 \%$ whereas a threefold increase from $h=0.1$ to $h=0.3$ leads to an erroneous detection of a volume increase of about $3 \%$. In contrast, the thresholding method (red cross-hairs) shows an underestimation of the soma volume that increases stepwise but maintains a constant volume over a range of heights. However, the stepwise decrease of the calculated volume and thus the determination of a constant volume over a certain range of heights results from the imprecision that occurs due to the rasterization of the sphere as shown by the investigation of a simulated scan with a tenfold resolution (red dots in Figure 10A). An increase in the height of the extension from $h=0.2$ to $h=0.6$ results in an erroneous detection of a volume decrease of about $16 \%$ for the low resolution simulation and of $32 \%$ in the high resolution simulation. We observed similar results when performing the same investigation on the objects depicted in Figure 9F and 9G with only slight differences in the amount of errors determined by the two methods.

We used $h r_{0}$ as threshold in these investigations. Note that the height of the processes of a live cell is much more difficult to determine due to the more complex and irregular shape and thus adds additional uncertainties to the determination of the soma volume.

Figure 10B shows the impact of various widths of the extension on the soma volume determination. As expected, the overestimation of the soma volume increases with increasing extension width $w 2 r_{0}$. A fourfold widening of the extension leads to an erroneous determination of a soma volume increase of $11 \%$. Since the height of the extension defines the threshold for the thresholding method the increasing width of the extension is not detected by this method. Thus it computes a constant volume under these conditions.

Figure 10C and 10D show the impact of a combined variation of the radii and the height of the extension. We investigated the radii $r_{x}(t)=r_{y}(t)=t^{1 / 2} r_{0}$ and $r_{z}(t)=r_{0} / t$ for $1 \leq t \leq 2$ and the fraction $h$ of the height $h r_{\mathrm{z}}(t)$ of the extension for $0.2 \leq h \leq 0.6$. Particularly when minor changes in shape were simulated, the BDA (Figure 10C) detects smaller erroneous volume changes compared with the thresholding method (Figure 10).

\section{Application to live cells}

We next applied the BDA to determine soma volumes in SICM recordings of live cells that exhibited both a much more irregular shape than the simulated objects as well as extensions that might be more difficult to distinguish 

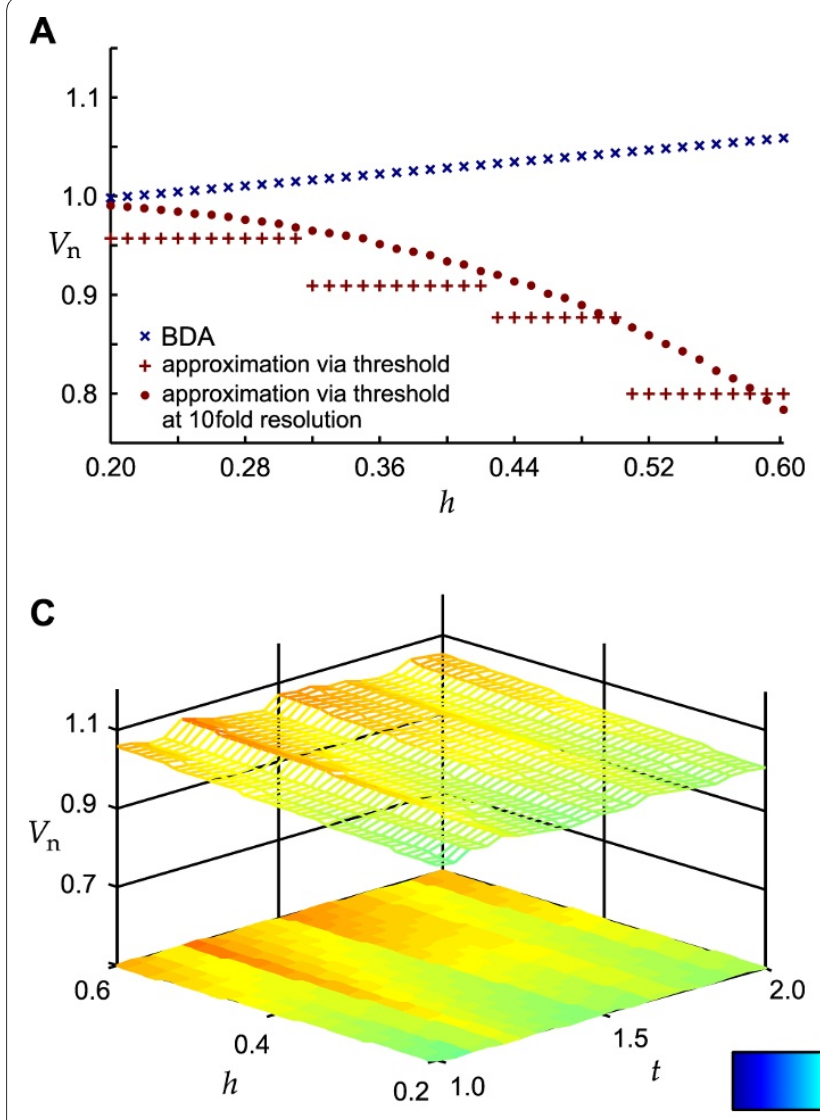

Figure 10 Simulating objects with varying geometrical parameters. A shows the volume normalized to the corresponding $V_{\text {sum }}$ determined by the boundary delimitation algorithm (BDA, blue crosses) and via thresholding (red dots) when simulating the height of the processes as $h r_{z}$ with $\Delta h$ $=0.01$. The stepwise decrease vanishes when the resolution increases (red cross-hairs). B shows the impact of the width of the processes by simulating their widths as $w 2 r_{y}$. Note that only the results for the BDA are shown. $C$ and $D$ show the volume normalized to the corresponding $V_{\text {sum }}$ when simulating objects with changing the processes' height (as in A) and the radii of the half-ellipsoid as $r_{x}=r_{y}=t^{1 / 2} r_{0}$ and $r_{z}=1 / t r_{0}$. The color coded area in the bottom indicates $V_{n}$ corresponding to the color bar plotted between $C$ and $D$. C shows the results for the BDA, D for thresholding. The corresponding steps of the parameters were $\Delta h=0.01$ and $\Delta t=0.05$.

from the cell soma. The corresponding data is available as Additional File 2. Figure 11 shows the results of the BDA applied to four different OPCs from rat brain. Note that the cells were positioned along the diagonal of the scan field in order to include as many details of the cell ramifications as possible. Whereas the cell somata depicted in Figure $11 \mathrm{Aa}$ and $\mathrm{Ba}$ approximate a circular shape the OPCs shown in Figure $11 \mathrm{Ca}$ and $\mathrm{Da}$ exhibited a more elongated cell soma that merged into one of the processes. The determination of the heading direction of the OPC shown in Figure 11 Aa selected the direction of the major process. Note that this might not be true when the fraction of the minor process that is located within the scan area notably exceeds the fraction of the major process. This might not impair the determination of the cell soma from a single scan but might have an impact when investigating the soma volume of a cell that migrates along the major process.
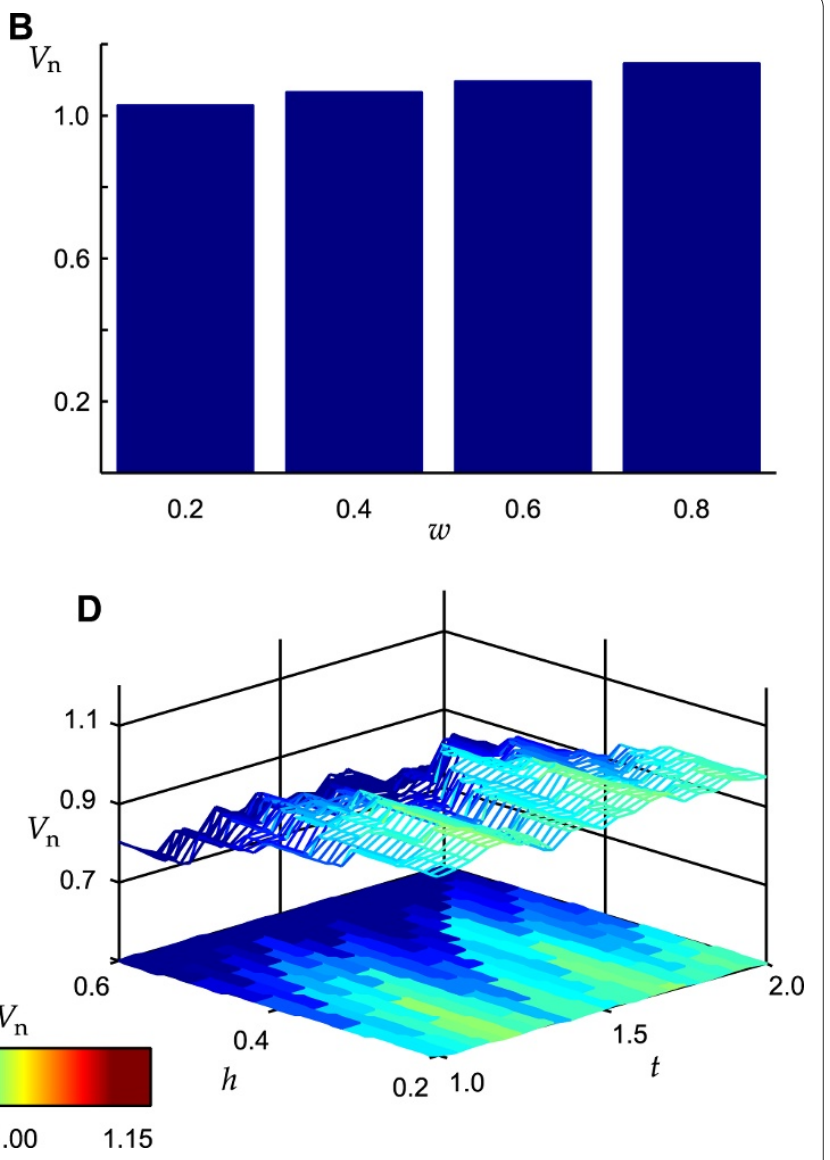

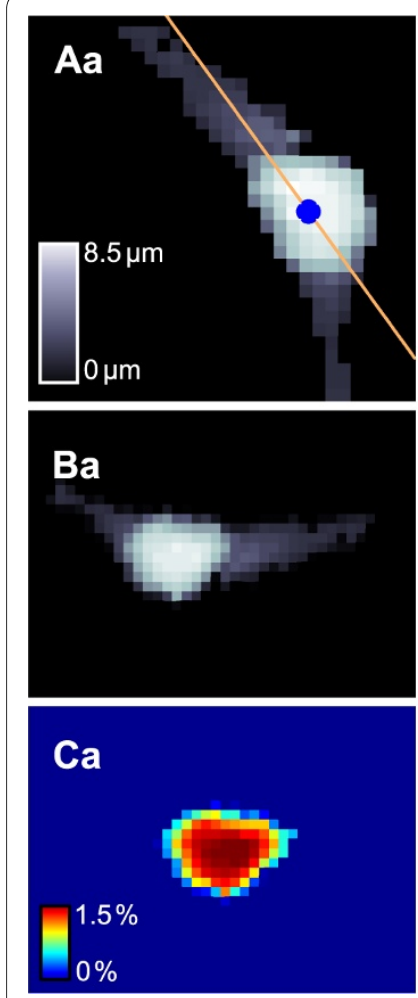
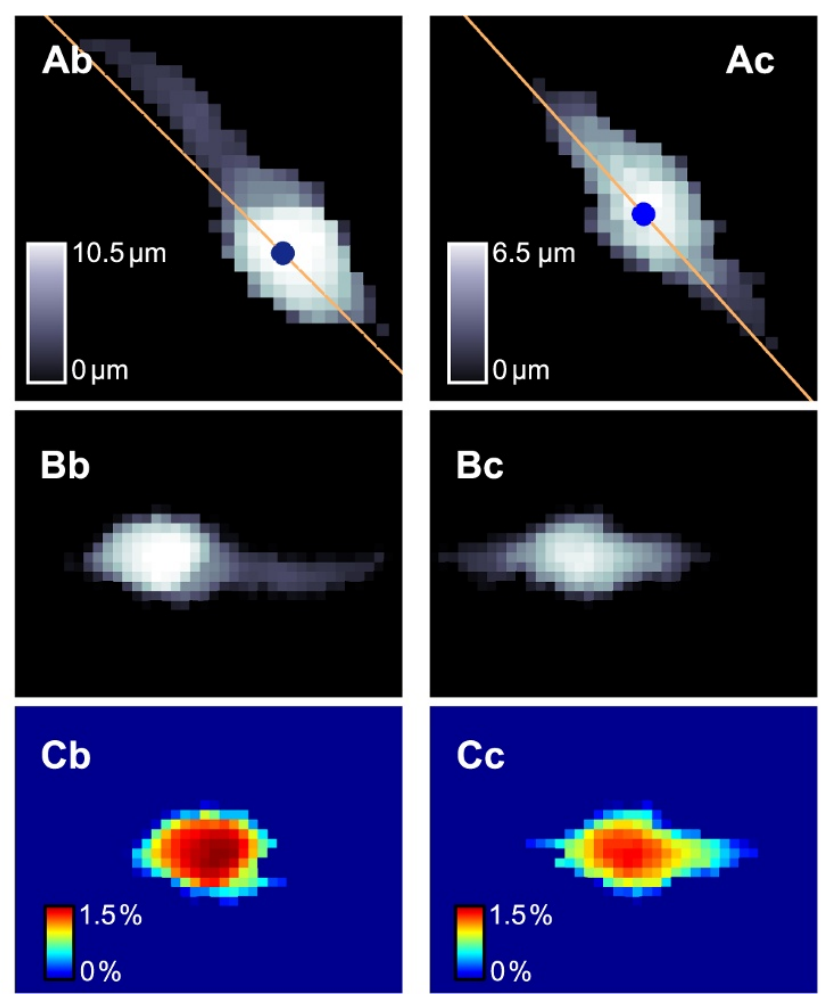
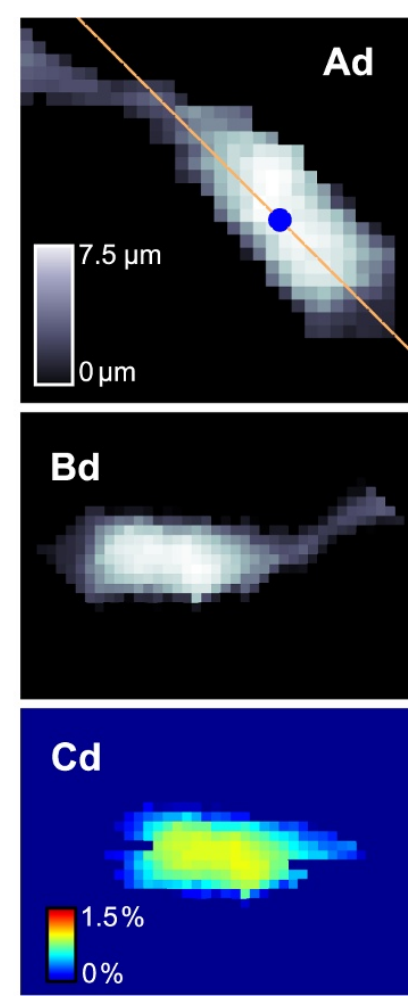

Figure 11 Application of the boundary delimitation algorithm to live cells. A: Top views of the original data of four different OPCs obtained by pulse-mode SICM in floating backstep configuration. Orange lines indicate $y\left(x, \theta_{h}\right)$, blue dots indicate $C_{90}$. B: Top views of the rotated, translated and interpolated data from the corresponding scans from A. The height of each scan and its corresponding rotated data is indicated by the color bars in A. C: Approximated soma area of the corresponding scans. The contribution of each pixel to the entire soma volume is indicated by the color bars at the bottom of each panel.

by the comparison of Figure $12 \mathrm{Ab}$ and $12 \mathrm{Bb}$. Note that it is known that migrating OPCs show an average velocity of $10 \mu \mathrm{m} / \mathrm{h}[28]$ and that migrating OPCs show notable changes in shape [27]. The detailed analysis of the parameters of the cell shape and soma shows that the cell swelled by approximately $29 \%$ and that this swelling was dominated by an increase in cell height whereas the length of the soma decreased. Most notably, this challenges the method to approximate the volume of a cell from light microscopic images by interpolation via the width and the length of its soma. This approximation, in contrast, would detect a slight cell shrinkage since the basal area covered by the cell soma was reduced as depicted in Figure 12E. The separate consideration of the frontal and rear soma volume by dividing the cell soma at the level of $C_{90}$ perpendicular to $y\left(x, \theta_{\mathrm{h}}\right)$ yields that the volume increase is dominated by an increase in the frontal volume (Figure 12E). Figure $12 \mathrm{~F}$ summarizes the changes in the lateral dimensions as well as the changes of area and volume between both scans.

\section{Conclusions}

We provide a new algorithm to approximate the basal area of the cell soma for volume determinations of bipolar cells undergoing temporal changes in shape. Simulations show that this method provides smaller errors in the detection of soma volume changes in moving cells than the method to separate soma areas from neurites via height thresholds $[16,19]$. We show that the algorithm can be applied successfully to detect soma volume changes of living oligodendrocyte precursor cells. Additionally we demonstrate that the approximation of the volume using two-dimensional data such as cell body length and width may lead to erroneous results.

\section{Methods}

\section{Scanning ion conductance microscopy}

Cells were obtained as described in [27] and images were acquired using a pulse mode SICM operating in floating backstep mode as detailed in [15,29]. Scans were performed in Leibovitz-15 medium using scanning probes with an access resistance of about $4 \mathrm{M} \Omega$. Scanning 


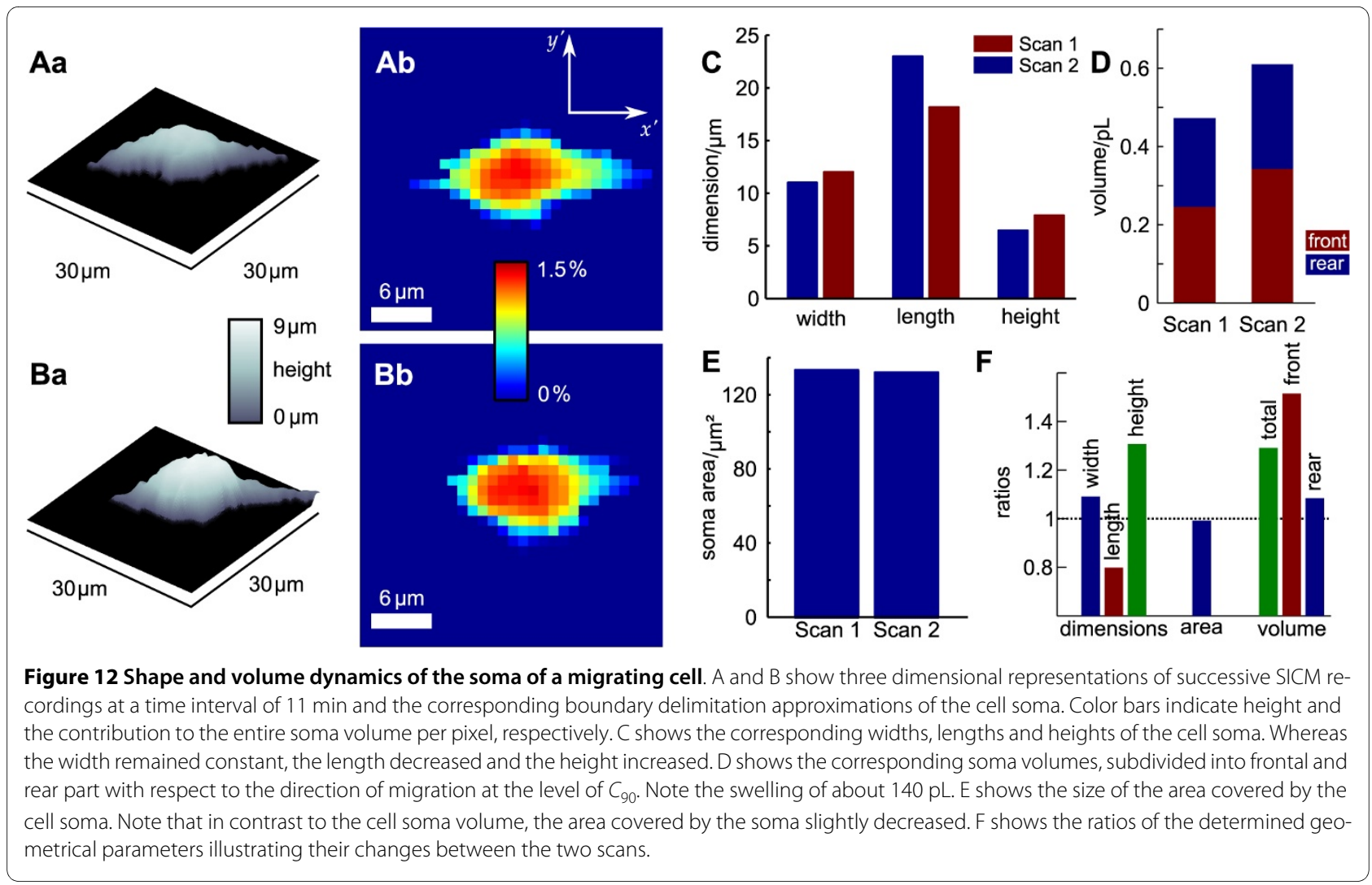

probes were filled with extracellular saline containing (in $\mathrm{mM}$ ): $\mathrm{NaCl} 110, \mathrm{KCl} 5.4, \mathrm{CaCl}_{2}$ 1.8, $\mathrm{MgCl}_{2}$ 0.8, HEPES 10, glucose 10. Step sizes were $1 \mu \mathrm{m}$ in lateral and $100 \mathrm{~nm}$ in vertical direction, frame acquisition time was about 10 minutes.

\section{Determination of the position of the nucleus}

Staining of the nucleus was performed by applying Hoechst 33342 dye $(20 \mu \mathrm{g} / \mathrm{mL}$, in Leibovitz-15 medium) for 5 minutes before the SICM measurement was started. Fluorescence images were obtained with a Zeiss Axiovert microscope at an epifluorescence wavelength of $365 \mathrm{~nm}$. The boundaries of the nucleus were obtained by applying the Sobel algorithm and thresholding. Centroids were calculated in the same manner as described above (equation (1)) but using a color value as threshold. The position of the scanning area within the light microscopic image was determined as described previously [19].

\section{Data processing}

SICM data was processed using Matlab (R2008a) including the Curve Fitting Toolbox [25]. Data shown were interpolated by cubic splines unless otherwise noted. All $z$-data were plane corrected if necessary and filtered with a threshold filter setting each $z$-value less than $1 \mu \mathrm{m}$ to 0 $\mu \mathrm{m}$ before the BDA was applied.

\section{Creation of half-ellipsoids}

The $z$-coordinates of the half-ellipsoids were computed as

$$
z(x, y)=r_{z} \Re\left(\sqrt{1-\frac{x^{2}}{r_{x}^{2}}-\frac{y^{2}}{r_{y}^{2}}}\right) .
$$

Here the operator $\mathrm{R}$ was defined to return the real part of a complex number $a+b \mathrm{i}$, hence $a=\mathrm{R}(a+b \mathrm{i})$ [22] and $r_{x}, r_{y}$ and $r_{z}$ denote the radii of the ellipsoid in the corresponding directions.

\section{Availability and requirements}

Project name: BDA - A boundary delimitation algorithm

Project homepage: https://sourceforge.net/projects/ behindlight/files/BDA/

Operating system(s): Platform independent

Programming language: Matlab

Other requirements: Matlab R2008a with Curve Fitting Toolbox

License: GNU-LGPL

Restrictions for commercial usage: Notify the corresponding author of this report and include a citation to this report in the accompanying documentation. 


\section{Additional material}

Additional file 1 This file is a zip archive including the Matlab code of the algorithm described above. To access the BDA extract (e.g. by using the unzip tool) the archive into a directory included in Matlab's path or, preferably, adjust Matlab's path. The archive consists of the following files (a detailed description is included in the respective file): cellDirection.m Determines the heading direction of a cell. See eqs. (2) and (3). CellFit.m Class performing the fitting procedure. See eqs. (8), (9) and (10), figs. 7 and 8 and section Approximation of the contour of a single data line. CellSomaVolumeCalculation.m Main class performing the BDA. It calls the respective procedures and class constructors and provides access to the data as described below. It calculates the dimensions of the rotated scan, see fig. 4 and eq. (4). centroid2 D.m Calculates the centroid of a given area, see eq. (1). getAreaAtHeight.m Reduces the $z$-coordinates of a set of pixels to boolean values indicating whether the $z$-coordinate exceeds a threshold. See section Approximation of the position of the nucleus. getInterpolZ.m Calculates the interpolated $z^{\prime}$-coordinate, see eqs. (6) and (7). getLine-

From Centroid.m Calculates the $x^{\prime}-, y^{\prime}$, , - and $z^{\prime}$-coordinates of a line parallel to the heading direction of the cell at a selectable distance from the $C_{90}$, therefore it performs the rotation and translation of the data. See eq. (5). SICMScan.m Class file for reading SICM data. To apply the BDA to scanning probe microscopical data stored in a text file consisting of three columns representing the $x-y$ - and $z$-coordinates use cell $=$ CellsomaVolumeCalculation ( '</path/to/data>') Here, </path/to/ data $>$ has to be substituted with the path and filename of the data to be investigated. After the BDA has finished the methods cell. plot, cell.plotRotated and cell.plotVolume are available to plot the raw data, the rotated and interpolated data and the contribution of each pixel to the cell soma volume, respectively (see fig. 11). The properties cell.Vol, cell.frontVol and cell.rearVol contain the total, front and rear volume, respectively (note that front and rear in this case represent $+x$ and $-x$-direction), cell . centroid and cell . angle contain the coordinates of $C_{-T}$ (as described above, we used $T=0.9$; the threshold can be varied in the properties section of CellsomaVolumeCalculation.m) and the heading direction of the cell, respectively.

Additional file $\mathbf{2}$ This file is a zip archive containing the data of the cells depicted in figs. 11 and 12 . Note that these data were processed as described in the Methods section. Files within the archive are named according to the figure label. Since the data shown in Figure $11 \mathrm{Ac}$ and Figure 12A originate from the same recording we included a soft link instead of a copy of the data.

\section{Authors' contributions}

$\mathrm{PH}$ and IDD designed the research. PH, RK and IDD wrote the manuscript. PH developed and coded the approximation method, KM performed the SICM measurements and the light microscopic determinations of the nucleus, prepared the cell culture and participated in the preparation of the manuscript. All authors read and approved the final manuscript.

\section{Acknowledgements}

Some of the equipment used was initially funded by the DFG-grant Schuh929/ 5-1 and we acknowledge the support of a part of the work by a fellowship of the Allgemeines Graduiertenkolleg der Ruhr-Universität Bochum to PH. We thank Heidrun Breuker-Siraj for help with the cell culture, Jonathan Coles, Gerd Hoffmann, Stefan A. Mann and Bernd Kind for critical comments on the manuscript as well as Rolf Heumann, Jan Meijer and the RUBION-team for continuous support.

\section{Author Details}

${ }^{1}$ Central Unit for lonbeams and Radionuclides (RUBION), Ruhr University of Bochum, D-44780 Bochum, Germany, 2Department of Molecular Neurobiochemistry, Ruhr University of Bochum, D-44780 Bochum, Germany and ${ }^{3}$ Department of Cardiovascular Physiology, University of Heidelberg, Heidelberg, Germany

Received: 10 March 2010 Accepted: 15 June 2010

Published: 15 June 2010

\section{References}

1. Lang F, Ritter M, Völkl H, Häussinger D: The biological significance of cell volume. Ren Physiol Biochem 1993, 16(12):48-65.

2. Wehner $F$, Olsen $H$, Tinel H, Kinne-Saffran $E$, Kinne RKH: Cell volume regulation: osmolytes, osmolyte transport, and signal transduction. Rev Physiol Biochem Pharmacol 2003, 148:1-80.

3. Dietzel I, Heinemann U, Hofmeier G, Lux HD: Transient changes in the size of the extracellular space in the sensorimotor cortex of cats in relation to stimulus-induced changes in potassium concentration. Exp Brain Res 1980, 40(4):432-9.

4. Dietzel I, Heinemann U, Lux HD: Relations between slow extracellular potential changes, glial potassium buffering, and electrolyte and cellular volume changes during neuronal hyperactivity in cat brain. Glia 1989, 2:25-44

5. Hall SK, Zhang J, Lieberman M: An early transient current is associated with hyposmotic swelling and volume regulation in embryonic chick cardiac myocytes. Exp Physiol 1997, 82:43-54.

6. Farinas J, Kneen M, Moore M, Verkman AS: Plasma membrane water permeability of cultured cells and epithelia measured by light microscopy with spatial filtering. J Gen Physiol 1997, 110(3):283-96.

7. Echevarria M, Verkman AS: Optical measurement of osmotic water transport in cultured cells. Role of glucose transporters. J Gen Physiol 1992, 99(4):573-89.

8. Kao HP, Verkman AS: Tracking of single fluorescent particles in three dimensions: use of cylindrical optics to encode particle position. Biophys J 1994, 67(3):1291-300.

9. Droste MS, Biel SS, Terstegen L, Wittern KP, Wenck H, Wepf R: Noninvasive measurement of cell volume changes by negative staining. J Biomed Opt 2005, 10(6):064017

10. Curl CL, Bellair CJ, Harris PJ, Allman BE, Roberts A, Nugent KA, Delbridge LM: Single cell volume measurement by quantitative phase microscopy (QPM): a case study of erythrocyte morphology. Cell Physiol Biochem 2006, 17(5-6):193-200.

11. Saadoun S, Papadopoulos MC, Hara-Chikuma M, Verkman AS: Impairment of angiogenesis and cell migration by targeted aquaporin1 gene disruption. Nature 2005, 434(7034):786-92.

12. Dieterich P, Klages R, Preuss R, Schwab A: Anomalous dynamics of cell migration. Proc Natl Acad Sci USA 2008, 105(2):459-63.

13. Satoh $H$, Delbridge LM, Blatter LA, Bers DM: Surface:volume relationship in cardiac myocytes studied with confocal microscopy and membrane capacitance measurements: species-dependence and developmental effects. Biophys J 1996, 70(3):1494-504.

14. Korchev Y, Gorelik J, Lab M, Sviderskaya E, Johnston C, Coombes C, Vodyanoy I, Edwards C: Cell volume measurement using scanning ion conductance microscopy. Biophys J 2000, 78:451-7.

15. Happel P, Hoffmann G, Mann S, Dietzel ID: Monitoring cell movements and volume changes with pulse-mode scanning ion conductance microscopy. J Microsc 2003, 212(Pt 2):144-51.

16. Mann SA, Versmold B, Marx R, Stahlhofen S, Dietzel ID, Heumann R, Berger $\mathrm{R}$ : Corticosteroids reverse cytokine-induced block of survival and differentiation of oligodendrocyte progenitor cells from rats. $J$ Neuroinflammation 2008, 5:39.

17. Schneider SW, Pagel P, Rotsch C, Danker T, Oberleithner H, Radmacher M, Schwab A: Volume dynamics in migrating epithelial cells measured with atomic force microscopy. Pflugers Arch 2000, 439(3):297-303.

18. Fabian A, Fortmann T, Dieterich P, Riethmüller C, Schön P, Mally S, Nilius B, Schwab A: TRPC1 channels regulate directionality of migrating cells. Pflugers Arch 2008, 457(2):475-84.

19. Mann SA, Meyer JW, Dietzel ID: Integration of a scanning ion conductance microscope into phase contrast optics and its application to the quantification of morphological parameters of selected cells. J Microsc 2006, 224(Pt 2):152-7.

20. Yunxu S, Danying L, Yanfang R, Dong H, Wanyun M: Three-dimensional structural changes in living hippocampal neurons imaged using magnetic AC mode atomic force microscopy. J Electron Microsc (Tokyo) 2006, 55(3):165-72.

21. Knuth DE: Two notes on notation. AMER MATH MONTHLY 99 1992, 5:403.

22. Graham RL, Knuth DE, Patashnik O: Concrete mathematics: a foundation for computer science Boston, MA, USA: Addison-Wesley Longman Publishing Co., Inc; 1994

23. Iverson KE: A programming language New York, NY, USA: John Wiley \& Sons, Inc; 1962. 
24. Sutherland IE, Sproull RF, Robert, Schumacker A: A characterization of ten hidden-surface algorithms. ACM Computing Surveys 1974, 6:1-55.

25. Mathworks: Curve Fitting Toolbox - Documentation. [http:// www.mathworks.com/access/helpdesk/help/toolbox/curvefit/

26. Mathworks: Fit model to data - MATLAB. [http://www.mathworks.com/ access/helpdesk/help/toolbox/curvefit/fit.html].

27. Happel P, Dietzel ID: Backstep scanning ion conductance microscopy as a tool for long term investigation of single living cells. J Nanobiotechnology 2009, 7:7.

28. Schmidt C, Ohlemeyer C, Labrakakis C, Walter T, Kettenmann H, Schnitzer $\mathrm{J}$ : Analysis of motile oligodendrocyte precursor cells in vitro and in brain slices. Glia 1997, 20(4):284-98.

29. Mann SA, Hoffmann G, Hengstenberg A, Schuhmann W, Dietzel ID: Pulsemode scanning ion conductance microscopy-a method to investigate cultured hippocampal cells. J Neurosci Methods 2002, 116(2):113-7.

doi: $10.1186 / 1471-2105-11-323$

Cite this article as: Happel et al., A boundary delimitation algorithm to approximate cell soma volumes of bipolar cells from topographical data obtained by scanning probe microscopy BMC Bioinformatics 2010, 11:323

Submit your next manuscript to BioMed Central and take full advantage of:

- Convenient online submission

- Thorough peer review

- No space constraints or color figure charges

- Immediate publication on acceptance

- Inclusion in PubMed, CAS, Scopus and Google Scholar

- Research which is freely available for redistribution

Submit your manuscript at www.biomedcentral.com/submit
Ciomed Central 